\title{
Los diversos efectos de las helmintiasis sobre la inflamación alérgica
}

\author{
Luis Caraballo \\ Instituto de Investigaciones Inmunológicas, Universidad de Cartagena, Cartagena de Indias, Colombia \\ Artículo de posesión para el ingreso como miembro de número a la \\ Academia Colombiana de Ciencias Exactas, Físicas y Naturales el 26 de abril de 2016
}

\begin{abstract}
Resumen
Las helmintiasis y las alergias son muy frecuentes en países del trópico, donde establecen múltiples interacciones. Los mecanismos de defensa contra estos parásitos son bastante parecidos a la respuesta inmunológica de tipo alérgico, principalmente porque ambos procesos tienen una importante participación de los anticuerpos tipo inmunoglobulina E (IgE). Pero también hay diferencias importantes, siendo una de ellas el grado de inmunomodulación, el cual es débil en la respuesta alérgica y fuerte en la antihelmíntica. Este tema tiene repercusiones en medicina porque algunas helmintiasis como la ascariasis (producida por Ascaris lumbricoides), son capaces de modificar el curso de las enfermedades alérgicas, en ocasiones disminuyendo su intensidad, por lo cual se ha propuesto que una de las razones del incremento de las alergias en el mundo es la disminución progresiva de las infecciones por helmintos. En esta revisión analizaremos varios de los efectos de las helmintiasis sobre la respuesta inmunológica alérgica y especularemos sobre su influencia en las tendencias internacionales de incremento de los problemas inflamatorios de origen inmunológico. (c) Acad. Colomb. Cienc. Ex. Fis. Nat. 2016.
\end{abstract}

Palabras clave: Helmintiasis, inmunomodulación, IgE, alergia, epidemiología, respuesta alérgica.

The various effects of helminthiases on allergic inflammation

\begin{abstract}
Helminth infections and allergies are very common in tropical countries, where they are linked by multiple interactions. Defense mechanisms against helminths are quite similar to the allergic immune response, mainly because both processes have a significant participation of antibodies such as immunoglobulin $\mathrm{E}$ (IgE). But there are also important differences, one being the degree of immunomodulation, which is weak in the allergic response and strong in anthelmintic immunity. This issue has implications in medicine because some helminth infections such as ascariasis (caused by Ascaris lumbricoides), are able to modify the course of allergic diseases, sometimes reducing their intensity and sometimes intensifying allergic symptoms. In this review we discuss some of the influence of helminthiases on the allergic immune response and speculate about its impact on the current international prevalence trends of inflammatory disorders. (c) Acad. Colomb. Cienc. Ex. Fis. Nat. 2016.
\end{abstract}

Key words: Helminthiases, immunomodulation, IgE, allergy, epidemiology, allergic response.

\section{Introducción}

Tanto las helmintiasis (infestaciones por parásitos helmintos) como las alergias (procesos inflamatorios de origen inmunológico) son muy comunes en las regiones tropicales, donde establecen múltiples interacciones. Al descubrirse los mecanismos de defensa contra estos parásitos se evidenció su parecido con la respuesta inmunológica de tipo alérgico, principalmente porque ambos procesos tienen una importante participación de los anticuerpos tipo inmunoglobulina $\mathrm{E}$ (IgE). Pero las semejanzas son más amplias e involucran otros componentes de la respuesta inmunológica; además, también hay diferencias importantes entre los dos procesos, siendo uno de ellos el grado de inmunomodulación, el cual es débil en la respuesta alérgica y fuerte en la antihelmíntica (Caraballo, 2013).

El tema es científicamente interesante por sus interrogantes sobre mecanismos básicos de las interacciones hospedero/ parásito, desde las más primitivas hasta las que hoy persisten después de una larga co-evolución. Además tiene repercusiones en medicina porque algunas helmintiasis como la ascariasis (producida por Ascaris lumbricoides), son capaces de modificar el curso de las enfermedades alérgicas, en ocasiones disminuyendo su intensidad, lo cual ha servido de sustento teórico para proponer que una de las razones del incremento de las alergias en el mundo es la disminución progresiva de las infecciones por helmintos. En esta revisión analizaremos varios de los efectos de las helmintiasis sobre la respuesta inmunológica alérgica y especularemos sobre su influencia en las tendencias internacionales de incremento de los problemas inflamatorios de origen inmunológico (Haahtela, et al., 2013).

\footnotetext{
Correspondencia:

Luis Caraballo, lcaraballog@unicartagena.edu.co

Recibido: 25 de marzo de 2016

Aceptado: 31 de mayo de 2016
} 


\section{Las interacciones entre helmintiasis y alergias}

Las helminthiasis más comunes son causadas por nematodos del suelo (Ascaris lumbricoides, Trichuris trichiura, Ancylostoma duodenalis, Necator americanus, Strongyloides stercoralis), filarias (Wuchereria bancrofti, Brugia malayi, Onchocerca volvulus) y trematodos (Schistosoma haematobium, Schistosoma mansoni, Schistosoma japonicum). La respuesta inmunológica durante esas infecciones varía dependiendo del tipo de parásito, su ciclo de vida, la edad del hospedero, la carga parasitaria y el poli parasitismo. Cerca de un billón de personas padecen ascariasis (Pullan, et al., 2014), un problema desatendido ("neglected") que se presenta principalmente en países pobres del trópico y cuyos graves efectos sobre la nutrición y el desarrollo de los niños son bien conocidos. La frecuencia y severidad de la ascariasis se ha reducido en los últimos 60 años, especialmente en países industrializados (Elliott and Weinstock, 2012, Feillet and Bach, 2004) y actualmente las formas más severas se concentran en algunas áreas rurales. En las zonas urbanas las condiciones higiénicas son mejores y se dan campañas regulares de desparasitación, lo que hace que la frecuencia y la intensidad sean menores. En Colombia, aunque no hay estudios destinados a comparar las frecuencias en estos dos ambientes, se observa que las helmintiasis son más comunes en zonas rurales (Agudelo-Lopez, et al., 2008, Carmona Fonseca Jaime 2009) que en las ciudades (Acevedo, et al., 2012, Londono, et al., 2009).

Por otro lado, una de cada tres personas sufre de alergias y la frecuencia de asma, rinitis y eczema atópico ha aumentado en los últimos años en la mayoría de los países donde se ha investigado (Dennis, et al., 2012, Mallol, et al., 2013, Pearce, et al., 2007). Estas enfermedades son muy frecuentes en las zonas urbanas, incluyendo aquellas de los países subdesarrollados (Endara, et al., 2015, Platts-Mills and Cooper, 2010, Rodriguez, et al., 2011), y merecen ser consideradas problemas de salud pública. Lo anterior significa que en una buena parte del planeta (la zona tórrida o tropical), donde habitan más de la mitad de los humanos, coexisten las parasitosis intestinales y las alergias, las primeras en una tendencia a disminuir y las segundas cada vez más frecuentes; reproduciendo en tiempo real lo que se supone ha sucedido en los países industrializados en las últimas 6 décadas (Figura 1). Los mecanismos por los cuales la urbanización se asocia con mayor prevalencia de alergias se desconocen, pero un factor podría ser la disminución de la carga parasitaria. Las alergias son enfermedades que resultan de la acción de múltiples factores ambientales sobre individuos genéticamente susceptibles. Las helmintiasis son apenas parte de esa influencia ambiental, la cual ocurre principalmente en el trópico. Por eso este escenario puede considerarse un experimento de la naturaleza cuyo análisis podría arrojar importante información sobre la patogénesis de problemas alérgicos (como el asma) y de las infecciones por helmintos (como la ascariasis).
Varias investigaciones en animales y humanos muestran que tanto la respuesta alérgica como la protectora contra helmintos tienen un fuerte componente Th2, con gran producción de IgE y citoquinas como IL-4, IL-5 e IL-13, la participación de células linfoides innatas (ILC), eosinófilos y células cebadas; además hiperplasia de células caliciformes con abundante producción de moco. Recientemente se ha descrito un importante papel de las células Tuft de la mucosa intestinal en la iniciación de la respuesta inmunitaria tipo 2 contra helmintos (Gerbe, et al., 2016), aunque su participación en la respuesta alérgica está por definirse.

Durante la infección, los helmintos secretan inmunomoduladores capaces de disminuir la respuesta protectora del hospedero induciendo linfocitos $\mathrm{T}$ y $\mathrm{B}$ reguladores (Tregs y Bregs) que incrementan la producción de IL-10 y TGF $\beta$; además promueven la síntesis de $\mathrm{IgG} / \mathrm{IgG} 4$ y alteran diversas vías de señalización intracelular que cambian el fenotipo de las células dendríticas a DC2 (las cuales promueven una respuesta Th2) y el de los macrófagos a M2, activados alternativamente y con menos capacidad proinflamatoria. Como resultado, la infección helmíntica se asocia a un importante grado de inmunosupresión que limita la capacidad del hospedero para adquirir inmunidad estéril.

Se supone que esto es producto de un largo camino de adaptación, en gran medida de los helmintos, durante el proceso evolutivo, lo cual ha permitido una relación parasitaria. De hecho, hay evidencias que sugieren este

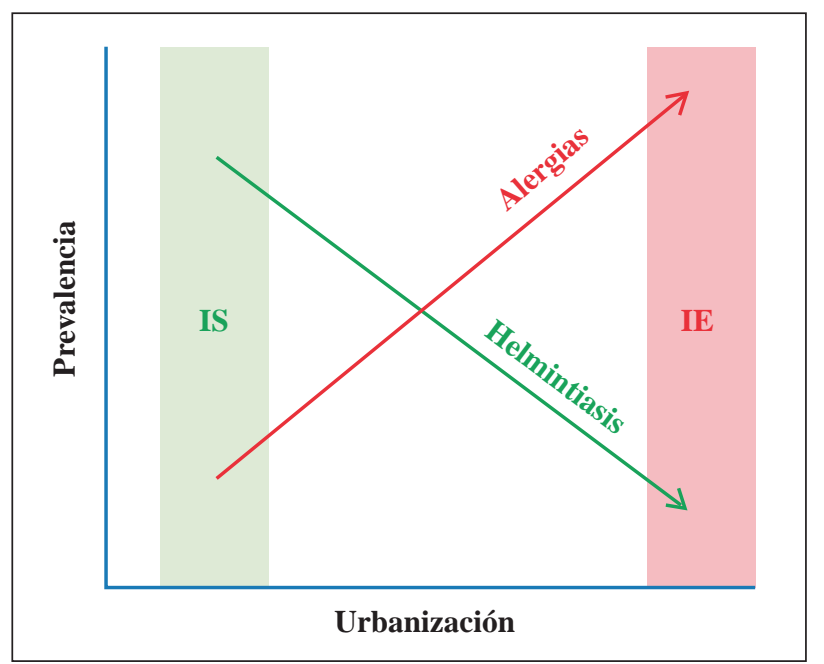

Figura 1. Representación hipotética del efecto del control de las helmintiasis sobre la prevalencia de las enfermedades alérgicas. La inmunosupresión (IS) alcanza su máximo cuando las helmintiasis son muy frecuentes y la carga de la infección es muy alta y la enfermedad severa; en este escenario, la prevalencia de síntomas alérgicos es baja. La inmunoestimulación (IE) prevalece cuando la frecuencia de las helmintiasis es más baja, lo que permite infecciones intermitentes y leves que aumentan los síntomas alérgicos. La hipótesis presupone que los cambios funcionan sobre una población genéticamente predispuesta a la alergia. 
tipo de adaptación a nivel molecular, por ejemplo, las cistatinas secretadas por filarias capaces de parasitar a los humanos tienen mayor efecto inmunosupresor que la cistatina del nematodo de vida libre Caenorhabditis elegans (Schierack, et al., 2003). Cuando ocurren infecciones severas por helmintos, lógicamente todos aquellos procesos inflamatorios derivados de una hiper respuesta inmunológica, como las enfermedades autoinmunes y las alergias, tienden a disminuir. Es importante mencionar que hasta el momento solo hay evidencias de esta influencia a nivel de los síntomas y no de la incepción de las enfermedades; esto último es un aspecto a considerar dado que en los sitios endémicos las parasitosis ocurren a edades tempranas y durante el embarazo (Cooper, et al., 2016, Maizels, et al., 2014, Smits and Akdis, 2014, Straubinger, et al., 2014).

Las interacciones helmintiasis/alergias son mutuas ya que hay evidencia de un efecto protector de las alergias contra la ascariasis (Lynch, et al., 1998); sin embargo, aquí no trataremos ese aspecto. Los efectos más visibles de la ascariasis sobre el asma se han identificado mediante estudios epidemiológicos con resultados aparentemente contradictorios porque en unos aparece como factor de riesgo, aumentando la frecuencia y la severidad del asma, mientras que en otros tiene efecto protector, disminuyendo la intensidad de los síntomas. Además, la ascariasis influye en el diagnóstico de las alergias porque reduce la especificidad de las pruebas que evalúan la IgE específica debido a la reactividad cruzada entre antígenos de Ascaris y ácaros. Finalmente, el impacto inmunomodulador de la ascariasis podría aprovecharse como terapia antiinflamatoria empleando moléculas inmunomoduladoras derivadas del parásito. A continuación revisaremos con más detalle estos temas.

\section{La ascariasis aumenta la intensidad de la respuesta alérgica mediada por IgE}

Esta sección tratará dos aspectos generales, a saber: los síntomas de alergia inducidos por la infección parasitaria y el aumento de dichos síntomas en las personas alérgicas infectadas por helmintos. Aunque posiblemente estén estrechamente relacionados, los analizaremos por separado con el fin de describir mejor las particularidades.

Históricamente, los primeros efectos relacionados con alergias observados en ascariasis fueron los síntomas de dificultad respiratoria o urticaria en niños infectados (Joubert, et al., 1979, Spillmann, 1975); sin embargo, estas y otras manifestaciones clínicas suceden también en helmintiasis como la strongyloidiasis, la hidatidosis (asociada a anafilaxis), la anisakiasis e inclusive infecciones por protozoarios, las cuales por sí mismas pueden inducir síntomas de alergia (Caraballo and Acevedo, 2011, Kolkhir, et al., 2016). Es importante tener en cuenta que esto ocurre en una minoría de la población infectada, lo cual podría tener, por lo menos, una explicación: La población que presenta síntomas alérgicos está genéticamente predispuesta a reaccionar de manera exagerada a la infección parasitaria, ya sea contra componentes propios del parásito o contra diversos componentes (población atópica); en ambos casos el grado de inmunosupresión concomitante no es suficiente para suprimir las manifestaciones de alergia, especialmente si la infección es leve.

Entonces, ¿por qué a nivel poblacional la gran mayoría de los parasitados por helmintos no presentan síntomas de alergia, siendo que las helmintiasis son fuertes potenciadores de la respuesta inmunológica Th2 y especialmente la síntesis de IgE? Una razón podría ser que la mayoría de la población es susceptible a los efectos inmunosupresores de los helmintos; estos últimos habrían evolucionado de tal forma que fueron seleccionados como parásitos aquellos capaces de generar inmunosupresión en los hospederos. Esta parte la trataremos más adelante, por ahora conviene concluir que las infecciones helmínticas por sí mismas son capaces de inducir síntomas de alergia, en un amplio rango que va, en creciente severidad, desde la ascariasis y strongyloidiasis hasta la hidatidosis y la anisakiasis. Por lo tanto, no sorprende que sean capaces de incrementar los síntomas en personas previamente alérgicas.

El efecto potenciador que ejerce la ascariasis sobre las alergias se puede detectar a nivel poblacional pues se ha observado repetidamente que la sensibilización contra alergenos de Ascaris se asocia con mayor prevalencia de asma (Ahumada, et al., 2015, Buendía, 2015, Hawlader, et al., 2014, Lynch, et al., 1997, Takeuchi, et al., 2015, Takeuchi, et al., 2008) incluso con mayor severidad (Buendía, 2015, Hunninghake, et al., 2007). Esto sucede tanto en zonas urbanas como rurales que tal vez comparten la condición de presentar infecciones leves (Endara, et al., 2015). También se manifiesta a nivel celular y molecular, induciendo una movilización de los componentes de la respuesta inmunológica Th2, especialmente de IgE específica contra antígenos del parásito y de reactividad cruzada. Una de las explicaciones más obvias de porqué la ascariasis incrementa los síntomas en las personas alérgicas es la gran reactividad cruzada entre los antígenos de Ascaris y los de los ácaros del polvo de habitación (Acevedo and Caraballo, 2011). Además, la infección por áscaris parece estimular la síntesis de IgE contra alergenos específicos de ácaros (Buendía, 2015, Zakzuk, 2014). Dado que estos últimos son los principales agentes causales de asma (especialmente en el Trópico) cualquier factor que incremente la respuesta alérgica contra sus componentes se convierte en un factor de riesgo para la enfermedad, especialmente si la población está expuesta de manera permanente a ambas fuentes.

Por lo menos 7 proteínas de Ascaris tienen reactividad cruzada con homólogas de los ácaros (Acevedo, et al., 2009b). De ellas, solo dos se han estudiado con detalle, la tropomiosina y la glutatión-transferasa/GST (Acevedo, et al., 2011, Acevedo, et al., 2013). También hay antígenos especie específicos; del lado del Ascaris la poli proteína ABA-1 (también conocida como Asc s 1), considerada un marcador 
de resistencia a la infección (McSharry, et al., 1999); y por parte de los ácaros los alergenos Blo t 5, Blo t 12 y Blo t 21 (Carvalho Kdos, et al., 2013, Zakzuk, et al., 2009). En la medida en que se ha avanzado en la caracterización de estas moléculas, se han planteado posibles mecanismos del porqué la sensibilización IgE contra Ascaris aumenta los síntomas de alergia. La tropomiosina, un pan alergeno, es la responsable de la mayor parte de la reactividad cruzada, y parece ser que la respuesta IgE contra ella genera el incremento de los síntomas en los alérgicos infectados (Ahumada, et al., 2015). Pero además, la ascariasis también aumenta los marcadores de severidad del asma, lo que indica que en las regiones tropicales (en contraste con las regiones temperadas) la sensibilización IgE a tropomiosina no es un mero hallazgo circunstancial sin efectos clínicos sino todo lo contrario, está asociado a la sintomatología y por lo tanto debe incluirse como prueba diagnóstica en las enfermedades alérgicas como el asma.

La influencia de la ascariasis sobre la prevalencia de asma podría tener importancia en las políticas de salud pública. Es bien conocido que las enfermedades parasitarias intestinales se pueden controlar con buenas condiciones de higiene y un adecuado desarrollo socio económico, lo cual no se ha logrado en muchos países muy a pesar de que son causantes de un alto grado de morbilidad en la población infantil. Sus efectos sobre el sistema inmunológico cada vez se documentan mejor, descubriendo una carga adicional que conllevan estas enfermedades desatendidas. Profundizar en el estudio de las interacciones helmintiasis/alergias es útil no solo para los países que actualmente sufren desnutrición y enfermedades parasitarias debido a la desidia política y el peso del subdesarrollo, sino para aquellos sectores industrializados que también tienen tasas elevadas de enfermedades alérgicas. Recientemente un grupo de investigadores de Bangladesh, considerando el efecto potenciador de la ascariasis sobre los síntomas de alergia (Ahumada, et al., 2015), ha propuesto que la reducción de la prevalencia de sibilancias en niños de ese país durante los últimos años está relacionada con las campañas sistemáticas de desparasitación (Takeuchi, et al., 2016). Es posible que tengan razón dado que actualmente el efecto más generalizado de la ascariasis sobre las alergias a nivel poblacional es el de aumentar su intensidad (Caraballo and Ahumada, 2016).

\section{La otra cara de la ascariasis: disminución de los síntomas de alergia}

Como se ha dicho, la infección por Ascaris activa simultáneamente mecanismos de inmunosupresión e inmunoestimulación en el hospedero (Acevedo and Caraballo, 2011). No ha sido fácil determinar cuándo a nivel clínico se nota una más que la otra. Podría decirse que un alto nivel de exposición, como en zonas rurales con malas condiciones higiénicas, sería un factor de riesgo para inmunosupresión, pero al parecer los componentes de resistencia y predisposición genéticos juegan también un papel importante
(Caraballo, 2013, Caraballo L, 2015). La inmunosupresión se ha detectado a nivel poblacional; se ha descrito que las infecciones severas por helmintos disminuyen la efectividad de las vacunas durante la infancia (Mkhize-Kwitshana and Mabaso, 2014, Salgame, et al., 2013). De igual manera varios estudios muestran baja frecuencia de alergias en zonas altamente parasitadas (Cooper, et al., 2004, Endara, et al., 2010, van den Biggelaar, et al., 2000) e hipo reactividad alérgica, especialmente en las pruebas cutáneas, en personas con helmintiasis (Rodrigues, et al., 2008, Stein, et al., 2016).

Por otro lado, las investigaciones básicas han descubierto diversas vías de inmunomodulación durante las infecciones por helmintos (Figura 2). Este aspecto es tal vez el más estudiado tanto a nivel epidemiológico como experimental, de donde proviene la mayoría de la información, algunas veces sorprendente (Osborne, et al., 2014, Reese, et al., 2014). La inmunosupresión inducida por los productos secretados por helmintos involucra diversas células y vías (Harnett, 2014), incluyendo el aumento de células T y B reguladoras (Hussaarts, et al., 2011, Metenou, et al., 2010, Zakzuk, 2016), la disminución de la activación de basófilos (Larson, et al., 2012) y en general altos niveles de IgE total y citoquinas como la IL-10 y TGF $\beta$ (Hagel, et al., 2011, Pitrez, et al., 2015). Otro aspecto importante es la producción de IgG (especialmente IgG4) contra componentes del parásito, observada en helmintiasis crónicas donde aparentemente es más fuerte la inmunomodulación (Figueiredo, et al., 2010). Este anticuerpo podría actuar como bloqueador inhibiendo la acción protectora y pro-inflamatoria de la IgE (Hagan, et al., 1991). De hecho, hemos observado que los niveles de IgG contra Ascaris fueron más altos en la población no alérgica que en un grupo de asmáticos alérgicos viviendo en el Trópico (Acevedo, et al., 2009a).

Ante el creciente aumento de las enfermedades de origen inmunológico a nivel mundial, es lógico que el efecto inmunosupresor de los helmintos despierte interés científico y comercial. Se ha evaluado la administración de helmintos no patógenos, que normalmente no infectan al humano, como tratamiento para procesos inflamatorios intestinales. Se ha llegado hasta utilizar patógenos como el Ancylostoma duodenale con el fin de disminuir la intensidad de rinitis alérgica. Los resultados de todas estas pruebas son contradictorios y han señalado que lo más práctico y ético es aislar las moléculas inmunomoduladoras para ser administradas a las dosis y presentaciones apropiadas.

De hecho, están en estudio varios componentes parasitarios con potencial utilidad como inmunomoduladores; aquí comentaremos más específicamente los de Ascaris. El análisis del genoma de Ascaris suum ha revelado que este parásito podría tener cerca de 10 componentes con actividad inmunomoduladora (Jex, et al., 2011), lo que podría ocurrir en Ascaris lumbricoides por la gran similitud entre estas dos especies. En A. lumbricoides se ha aislado, purificado y evaluado, la molécula AlCPI, una cistatina inhibidora de 


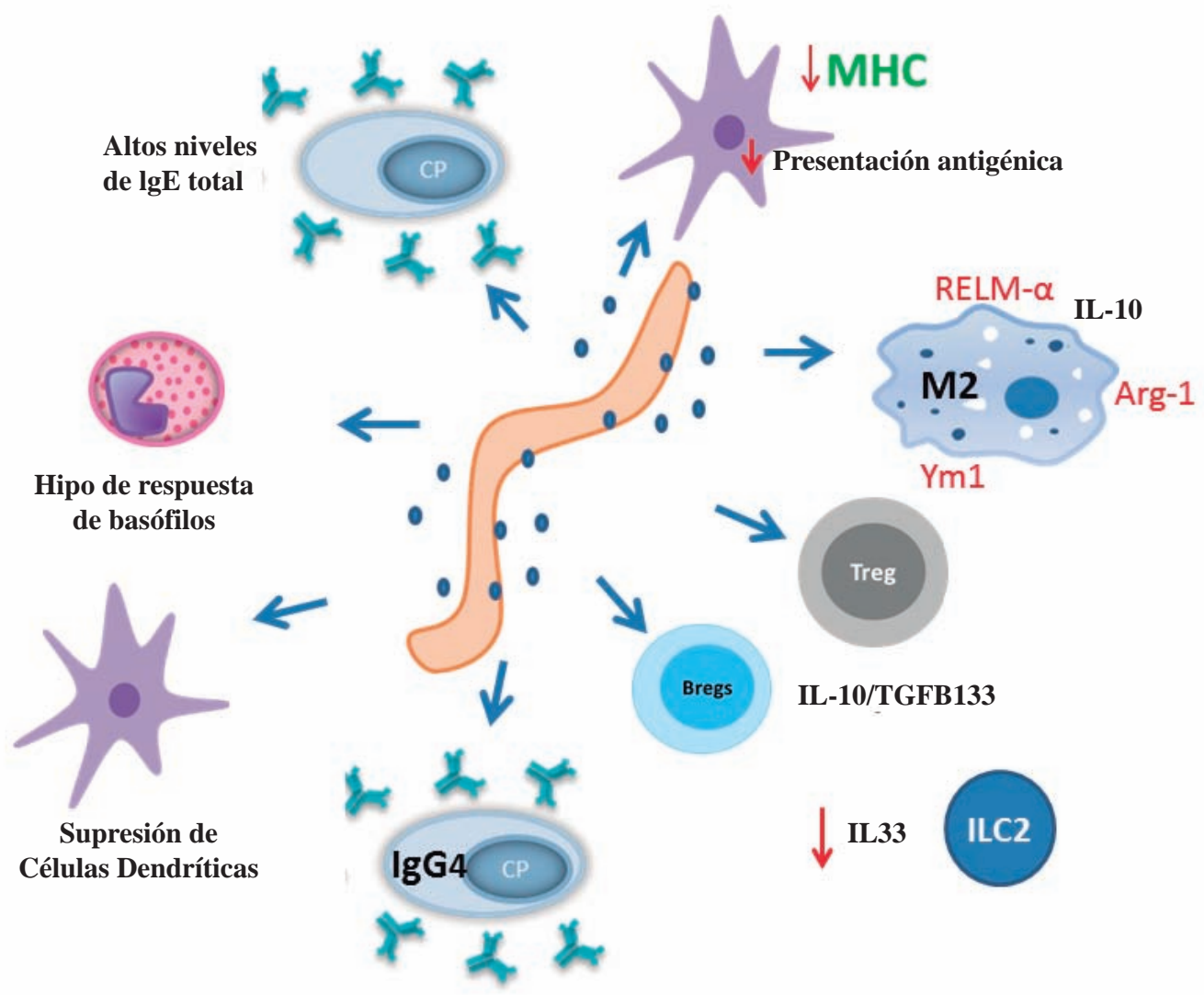

Figura 2. Mecanismos de inmunomodulación por helmintos. Los helmintos secretan y/o excretan diversas substancias con efectos inmunomoduladores, algunos esquematizados en la figura. Los altos niveles de IgE total característicos de las helmintiasis podrían ocupar los receptores Fc de los basófilos, compitiendo con la IgE específica y disminuyendo la reactividad de estas células hacia los alergenos. Entre los mecanismos más reproducibles están el aumento de las células reguladoras ( $\mathrm{T}$ y B) y la activación de los macrófagos por la vía alterna, lo cual disminuye su capacidad inflamatoria. Algunos componentes afectan la expresión de las moléculas MHC clase II y adicionalmente limitan la actividad del proteasoma, con el resultado final de una disminución de la presentación antigénica. Uno de los efectos más interesantes es la supresión de las células dendríticas, la cual, asociada a una disminución de la síntesis de IL33, afecta las fases iniciales de la respuesta inmunológica comprometiendo también a las células linfoides innatas (ILC).

proteasas (Coronado S, 2016) y en A. suum se detectado también actividad antiinflamatoria en la proteína excretada/ secretada PAS1 (Antunes, et al., 2015).

\section{Resumen y conclusiones}

Hace algunos años parecía que los resultados contradictorios de los estudios epidemiológicos sobre la relación helmintiasis/alergia no eran más que el resultado de diferentes diseños metodológicos. Hoy vemos claramente que esas investigaciones de corte transversal detectaban lo ocurrido en condiciones climáticas, higiénicas y poblacionales precisas, que a su vez reflejaban la gran variabilidad a la exposición a los parásitos que existe en la actualidad. Como se sabe, el desarrollo económico y social de los países es desigual, con diferencias importantes aún entre los subdesarrollados, especialmente en cuanto a la dinámica y calidad del proceso de urbanización. No es de extrañar entonces que estudios que evalúan condiciones extremas de exposición a los parásitos, es decir, de baja y alta endemicidad, muestren resultados que en lugar de ser contradictorios nos están indicando que el nivel de exposición, así como el grado de severidad y cronicidad de las infecciones por helmintos, son determinantes para definir si predomina la inmunoestimulación sobre la inmunomodulación (Figura 3).

Un buen número de estudios experimentales, tanto en animales como en humanos sustentan cada vez más que las helmintiasis, incluyendo la ascariasis, inducen mecanismos inmunoreguladores (estimulación y supresión) que modifican la respuesta inmunológica de los hospederos. Esto tiene coherencia con la teoría de la evolución mediante selección natural ya que combina una fuerte respuesta protectora antiparasitaria con la suficiente tolerancia para que haya algún grado de parasitismo. Los escasos estudios de cohorte de nacimiento actualmente en curso en países tropicales (Acevedo, et al., 2012) tienen la posibilidad de profundizar en estos aspectos. De igual manera, la disponibilidad de moléculas recombinantes, tanto alergénicas como inmunosupresoras, permitirá precisar mejor los mecanismos moleculares de la inmunomodulación. 


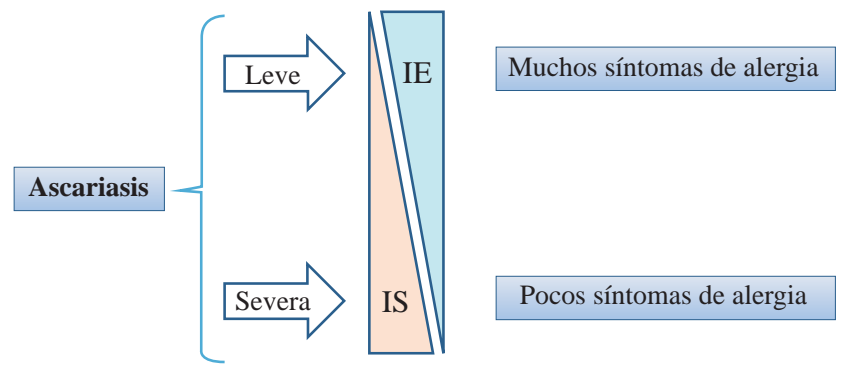

Figura 3. Resumen esquemático de las dos posibilidades principales en la interacción de helmintiasis (por ejemplo, la ascariasis) con la respuesta alérgica y sus síntomas en una población del trópico, expuesta simultánea y perennemente tanto a los helmintos como a los alergenos de ácaros. Las infecciones leves inducen inmunoestimulación (IE) y mantienen o aumentan los síntomas de alergia, mientras que las severas inducen inmunosupresión (IS), la cual disminuye los síntomas. Nótese que ambos fenómenos suceden siempre pero con diferente intensidad, lo cual establece un gradiente que afectará los resultados de los estudios epidemiológicos. La figura no incluye los aspectos genéticos tanto del parásito como del hospedero.

Por ahora solo podemos afirmar que a pesar de tanta política adversa al desarrollo, este se ha dado en cierta medida y las condiciones higiénicas han mejorado en algunos sectores; de manera que hoy la exposición leve/ moderada a Ascaris supera en frecuencia a los niveles severos confinados a zonas rurales. De allí se deduce que actualmente el efecto inmunomodulador más frecuente de la ascariasis es la inmunoestimulación. La mayoría de los países industrializados ya pasaron por estas etapas y su prevalencia de enfermedades alérgicas es actualmente alta. Qué tanto tiene que ver esto con la ausencia de los efectos inmunosupresores de las helmintiasis está por definirse, pero el hecho de que en países menos desarrollados del trópico, donde todavía hay helmintiasis, la prevalencia de alergias sea similar o más elevada, sugiere que podría estar relacionada.

Tal vez lo que está sucediendo es que las helmintiasis ejercen su efecto estimulante en la población más susceptible, es decir la que está predispuesta genéticamente a responder de manera exagerada a ciertos estímulos ambientales: la población potencialmente alérgica. De allí que las cifras de prevalencia de alergias hayan llegado a una meseta en los últimos años, resistiendo los variados factores de riesgo existentes, entre los cuales las helmintiasis son apenas una parte. Podría decirse que en el futuro las alergias aumentarán en aquellas regiones actualmente muy expuestas a las helmintiasis en la medida en que las condiciones higiénicas mejoren. En las demás un aumento en frecuencia requeriría de cambios en la composición genética de la población.

\section{Agradecimientos}

Las investigaciones del autor que sustentan esta revisión fueron financiadas por Colciencias, contratos 406-2011 y 590-2013 y la Universidad de Cartagena (Plan de fortalecimiento de Grupo).

\section{Conflicto de intereses}

El autor declara no tener conflicto de intereses.

\section{Bibliografía}

Acevedo, N. \& Caraballo, L. (2011). IgE cross-reactivity between Ascaris lumbricoides and mite allergens: possible influences on allergic sensitization and asthma. Parasite Immunol 33: 309-21.

Acevedo, N., Erler, A., Briza, P., Puccio, F., Ferreira, F. \& Caraballo, L. (2011). Allergenicity of Ascaris lumbricoides tropomyosin and IgE sensitization among asthmatic patients in a tropical environment. Int Arch Allergy Immunol 154: 195-206.

Acevedo, N., Mercado, D., Vergara, C., Sánchez, J., Kennedy, M. W., Jiménez, S., Fernández, A. M., Gutiérrez, M., Puerta, L. \& Caraballo, L. (2009a). Association between total immunoglobulin E and antibody responses to naturally acquired Ascaris lumbricoides infection and polymorphisms of immune system-related LIG4, TNFSF13B and IRS2 genes. Clin Exp Immunol 157: 282-90.

Acevedo, N., Mohr, J., Zakzuk, J., Samonig, M., Briza, P., Erler, A., Pomes, A., Huber, C. G., Ferreira, F. \& Caraballo, L. (2013). Proteomic and immunochemical characterization of glutathione transferase as a new allergen of the nematode Ascaris lumbricoides. PLoS One 8: e78353.

Acevedo, N., Sanchez, J., Erler, A., Mercado, D., Briza, P., Kennedy, M., Fernandez, A., Gutierrez, M., Chua, K. Y., Cheong, N., Jimenez, S., Puerta, L. \& Caraballo, L. (2009b). IgE cross-reactivity between Ascaris and domestic mite allergens: the role of tropomyosin and the nematode polyprotein ABA-1. Allergy 64: 1635-43.

Acevedo, N., Sanchez, J., Zakzuk, J., Bornacelly, A., Quiroz, C., Alvarez, A., Puello, M., Mendoza, K., Martinez, D., Mercado, D., Jimenez, S. \& Caraballo, L. (2012). Particular characteristics of allergic symptoms in tropical environments: follow up to 24 months in the FRAAT birth cohort study. BMC Pulm Med 12: 13.

Agudelo-Lopez, S., Gomez-Rodriguez, L., Coronado, X., Orozco, A., Valencia-Gutierrez, C. A., Restrepo-Betancur, L. F., Galvis-Gomez, L. A. \& Botero-Palacio, L. E. (2008). [Prevalence of intestinal parasitism and associated factors in a village on the Colombian Atlantic Coast]. Rev Salud Publica (Bogota) 10: 633-42.

Ahumada, V., Garcia, E., Dennis, R., Rojas, M. X., Rondon, M. A., Perez, A., Penaranda, A., Barragan, A. M., Jimenez, S., Kennedy, M. W. \& Caraballo, L. (2015). IgE responses to Ascaris and mite tropomyosins are risk factors for asthma. Clin Exp Allergy 45: 1189-1200.

Antunes, M. F., Titz, T. O., Batista, I. F., Marques-Porto, R., Oliveira, C. F., Alves de Araujo, C. A. \& Macedo-Soares, M. F. (2015). Immunosuppressive PAS-1 is an excretory/ secretory protein released by larval and adult worms of the ascarid nematode Ascaris suum. J Helminthol 89: 367-74.

Buendía, E. (2015). The IgE response to Ascaris molecular components is associated with clinical indicators of asthma severity. WAO Journal 8: 8. 
Caraballo, L. (2013). Ascaris and Allergy. In Ascaris: the neglected parasite (ed. C. V. Holland), pp. 21-50. Elsevier.

Caraballo, L. \& Acevedo, N. (2011). New allergens of relevance in tropical regions: the impact of Ascaris lumbricoides infections. WAO Journal 4: 77-84.

Caraballo, L. \& Ahumada, V. (2016). Reply to 'Comments on IgE responses to Ascaris and mite tropomyosins are risk factors for asthma'. Clin Exp Allergy 46: 181.

Caraballo L, A. N., Buendía E. (2015). Human Ascariasis Increases the Allergic Response and Allergic Symptoms. Curr Trop Med Rep 2: 224-232.

Carmona Fonseca Jaime, U. R., Correa Botero Adriana María (2009). Parasitosis intestinal en niños de zonas palúdicas de Antioquia (Colombia). IATREIA 22: 28-46.

Carvalho Kdos, A., de Melo-Neto, O. P., Magalhaes, F. B., Ponte, J. C., Felipe, F. A., dos Santos, M. C., dos Santos Lima, G., Cruz, A. A., Pinheiro, C. S., Pontes-de-Carvalho, L. C. \& Alcantara-Neves, N. M. (2013). Blomia tropicalis Blo t 5 and Blo t 21 recombinant allergens might confer higher specificity to serodiagnostic assays than whole mite extract. BMC Immunol 14: 11.

Cooper, P. J., Chico, M. E., Amorim, L. D., Sandoval, C., Vaca, M., Strina, A., Campos, A. C., Rodrigues, L. C., Barreto, M. L. \& Strachan, D. P. (2016). Effects of maternal geohelminth infections on allergy in early childhood. $J$ Allergy Clin Immunol 137: 899-906 e2.

Cooper, P. J., Chico, M. E., Rodrigues, L. C., Strachan, D. P., Anderson, H. R., Rodriguez, E. A., Gaus, D. P. \& Griffin, G. E. (2004). Risk factors for atopy among school children in a rural area of Latin America. Clin Exp Allergy 34: 845-52.

Coronado S, e. a. (2016). A recombinant cystatin from Ascaris lumbricoides has immunomodulatory effects. Journal of Allergy and Clinical Immunology 137: AB228.

Dennis, R. J., Caraballo, L., Garcia, E., Rojas, M. X., Rondon, M. A., Perez, A., Aristizabal, G., Penaranda, A., Barragan, A. M., Ahumada, V. \& Jimenez, S. (2012). Prevalence of asthma and other allergic conditions in Colombia 2009-2010: a cross-sectional study. BMC Pulm Med 12: 17 .

Elliott, D. E. \& Weinstock, J. V. (2012). Where are we on worms? Curr Opin Gastroenterol 28: 551-6.

Endara, P., Vaca, M., Chico, M. E., Erazo, S., Oviedo, G., Quinzo, I., Rodriguez, A., Lovato, R., Moncayo, A. L., Barreto, M. L., Rodrigues, L. C. \& Cooper, P. J. (2010). Long-term periodic anthelmintic treatments are associated with increased allergen skin reactivity. Clin Exp Allergy 40: 1669-77.

Endara, P., Vaca, M., Platts-Mills, T. A., Workman, L., Chico, M. E., Barreto, M. L., Rodrigues, L. C. \& Cooper, P. J. (2015). Effect of urban vs. rural residence on the association between atopy and wheeze in Latin America: findings from a case-control analysis. Clin Exp Allergy 45: 438-47.

Feillet, H. \& Bach, J. F. (2004). Increased incidence of inflammatory bowel disease: the price of the decline of infectious burden? Curr Opin Gastroenterol 20: 560-4.
Figueiredo, C. A., Barreto, M. L., Rodrigues, L. C., Cooper, P. J., Silva, N. B., Amorim, L. D. \& Alcantara-Neves, N. M. (2010). Chronic intestinal helminth infections are associated with immune hyporesponsiveness and induction of a regulatory network. Infect Immun 78: 3160-7.

Gerbe, F., Sidot, E., Smyth, D. J., Ohmoto, M., Matsumoto, I., Dardalhon, V., Cesses, P., Garnier, L., Pouzolles, M., Brulin, B., Bruschi, M., Harcus, Y., Zimmermann, V. S., Taylor, N., Maizels, R. M. \& Jay, P. (2016). Intestinal epithelial tuft cells initiate type 2 mucosal immunity to helminth parasites. Nature 529: 226-30.

Haahtela, T., Holgate, S., Pawankar, R., Akdis, C. A., Benjaponpitak, S., Caraballo, L., Demain, J., Portnoy, J., von Hertzen, L., Change, W. A. O. S. C. o. C. \& Biodiversity (2013). The biodiversity hypothesis and allergic disease: world allergy organization position statement. World Allergy Organ J 6: 3 .

Hagan, P., Blumenthal, U. J., Dunn, D., Simpson, A. J. \& Wilkins, H. A. (1991). Human IgE, IgG4 and resistance to reinfection with Schistosoma haematobium. Nature 349: 243-5.

Hagel, I., Cabrera, M., Puccio, F., Santaella, C., Buvat, E., Infante, B., Zabala, M., Cordero, R. \& Di Prisco, M. C. (2011). Co-infection with Ascaris lumbricoides modulates protective immune responses against Giardia duodenalis in school Venezuelan rural children. Acta Trop 117: 189-95.

Harnett, W. (2014). Secretory products of helminth parasites as immunomodulators. Mol Biochem Parasitol 195: 130-6.

Hawlader, M. D., Ma, E., Noguchi, E., Itoh, M., Arifeen, S. E., Persson, L. A., Moore, S. E., Raqib, R. \& Wagatsuma, Y. (2014). Ascaris lumbricoids Infection as a Risk Factor for Asthma and Atopy in Rural Bangladeshi Children. Trop Med Health 42: 77-85.

Hunninghake, G. M., Soto-Quiros, M. E., Avila, L., Ly, N. P., Liang, C., Sylvia, J. S., Klanderman, B. J., Silverman, E. K. \& Celedon, J. C. (2007). Sensitization to Ascaris lumbricoides and severity of childhood asthma in Costa Rica. J Allergy Clin Immunol 119: 654-61.

Hussaarts, L., van der Vlugt, L. E., Yazdanbakhsh, M. \& Smits, H. H. (2011). Regulatory B-cell induction by helminths: implications for allergic disease. J Allergy Clin Immunol 128: 733-9.

Jex, A. R., Liu, S., Li, B., Young, N. D., Hall, R. S., Li, Y., Yang, L., Zeng, N., Xu, X., Xiong, Z., Chen, F., Wu, X., Zhang, G., Fang, X., Kang, Y., Anderson, G. A., Harris, T. W., Campbell, B. E., Vlaminck, J., Wang, T., Cantacessi, C., Schwarz, E. M., Ranganathan, S., Geldhof, P., Nejsum, P., Sternberg, P. W., Yang, H., Wang, J., Wang, J. \& Gasser, R. B. (2011). Ascaris suum draft genome. Nature 479: 529-33.

Joubert, J. R., de Klerk, H. C. \& Malan, C. (1979). Ascaris lumbricoides and allergic asthma: A new perspective. S Afr Med J 56: 599-602.

Kolkhir, P., Balakirski, G., Merk, H. F., Olisova, O. \& Maurer, M. (2016). Chronic spontaneous urticaria and internal parasites - a systematic review. Allergy 71: 308-22. 
Larson, D., Cooper, P. J., Hubner, M. P., Reyes, J., Vaca, M., Chico, M., Kong, H. H. \& Mitre, E. (2012). Helminth infection is associated with decreased basophil responsiveness in human beings. J Allergy Clin Immunol 130: $270-2$.

Londono, A. L., Mejia, S. \& Gomez-Marin, J. E. (2009). [Prevalence and risk factors associated with intestinal parasitism in preschool children from the urban area of Calarca, Colombia]. Rev Salud Publica (Bogota) 11: 72-81.

Lynch, N. R., Hagel, I. A., Palenque, M. E., Di Prisco, M. C., Escudero, J. E., Corao, L. A., Sandia, J. A., Ferreira, L. J., Botto, C., Perez, M. \& Le Souef, P. N. (1998). Relationship between helminthic infection and IgE response in atopic and nonatopic children in a tropical environment. J Allergy Clin Immunol 101: 217-21.

Lynch, N. R., Palenque, M., Hagel, I. \& DiPrisco, M. C. (1997). Clinical improvement of asthma after anthelminthic treatment in a tropical situation. Am J Respir Crit Care Med 156: $50-4$.

Maizels, R. M., McSorley, H. J. \& Smyth, D. J. (2014). Helminths in the hygiene hypothesis: sooner or later? Clin Exp Immunol 177: 38-46.

Mallol, J., Crane, J., von Mutius, E., Odhiambo, J., Keil, U., Stewart, A. \& Group, I. P. T. S. (2013). The International Study of Asthma and Allergies in Childhood (ISAAC) Phase Three: a global synthesis. Allergol Immunopathol (Madr) 41: 73-85.

McSharry, C., Xia, Y., Holland, C. V. \& Kennedy, M. W. (1999). Natural immunity to Ascaris lumbricoides associated with immunoglobulin E antibody to ABA-1 allergen and inflammation indicators in children. Infect Immun 67: 484-9.

Metenou, S., Dembele, B., Konate, S., Dolo, H., Coulibaly, S. Y., Coulibaly, Y. I., Diallo, A. A., Soumaoro, L., Coulibaly, M. E., Sanogo, D., Doumbia, S. S., Traore, S. F., Mahanty, S., Klion, A. \& Nutman, T. B. (2010). At homeostasis filarial infections have expanded adaptive $\mathrm{T}$ regulatory but not classical Th2 cells. J Immunol 184: 5375-82.

Mkhize-Kwitshana, Z. L. \& Mabaso, M. L. (2014). The neglected triple disease burden and interaction of helminths, HIV and tuberculosis: an opportunity for integrated action in South Africa. S Afr Med J 104: 258, 259.

Osborne, L. C., Monticelli, L. A., Nice, T. J., Sutherland, T. E., Siracusa, M. C., Hepworth, M. R., Tomov, V. T., Kobuley, D., Tran, S. V., Bittinger, K., Bailey, A. G., Laughlin, A. L., Boucher, J. L., Wherry, E. J., Bushman, F. D., Allen, J. E., Virgin, H. W. \& Artis, D. (2014). Coinfection. Virushelminth coinfection reveals a microbiota-independent mechanism of immunomodulation. Science 345: 578-82.

Pearce, N., Ait-Khaled, N., Beasley, R., Mallol, J., Keil, U., Mitchell, E. \& Robertson, C. (2007). Worldwide trends in the prevalence of asthma symptoms: phase III of the International Study of Asthma and Allergies in Childhood (ISAAC). Thorax 62: 758-66.
Pitrez, P. M., Gualdi, L. P., Barbosa, G. L., Sudbrack, S., Ponzi, D., Cao, R. G., Silva, A. C., Machado, D. C., Jones, M. H., Stein, R. T. \& Graeff-Teixeira, C. (2015). Effect of different helminth extracts on the development of asthma in mice: The influence of early-life exposure and the role of IL-10 response. Exp Parasitol 156: 95-103.

Platts-Mills, T. A. \& Cooper, P. J. (2010). Differences in asthma between rural and urban communities in South Africa and other developing countries. J Allergy Clin Immunol 125: 106-7.

Pullan, R. L., Smith, J. L., Jasrasaria, R. \& Brooker, S. J. (2014). Global numbers of infection and disease burden of soil transmitted helminth infections in 2010. Parasit Vectors 7: 37.

Reese, T. A., Wakeman, B. S., Choi, H. S., Hufford, M. M., Huang, S. C., Zhang, X., Buck, M. D., Jezewski, A., Kambal, A., Liu, C. Y., Goel, G., Murray, P. J., Xavier, R. J., Kaplan, M. H., Renne, R., Speck, S. H., Artyomov, M. N., Pearce, E. J. \& Virgin, H. W. (2014). Helminth infection reactivates latent gamma-herpesvirus via cytokine competition at a viral promoter. Science 345: 573-7.

Rodrigues, L. C., Newcombe, P. J., Cunha, S. S., AlcantaraNeves, N. M., Genser, B., Cruz, A. A., Simoes, S. M., Fiaccone, R., Amorim, L., Cooper, P. J., Barreto, M. L., Social Change, A. \& Allergy in Latin, A. (2008). Early infection with Trichuris trichiura and allergen skin test reactivity in later childhood. Clin Exp Allergy 38: 1769-77.

Rodriguez, A., Vaca, M., Oviedo, G., Erazo, S., Chico, M. E., Teles, C., Barreto, M. L., Rodrigues, L. C. \& Cooper, P. J. (2011). Urbanisation is associated with prevalence of childhood asthma in diverse, small rural communities in Ecuador. Thorax 66: 1043-50.

Salgame, P., Yap, G. S. \& Gause, W. C. (2013). Effect of helminthinduced immunity on infections with microbial pathogens. Nat Immunol 14: 1118-26.

Schierack, P., Lucius, R., Sonnenburg, B., Schilling, K. \& Hartmann, S. (2003). Parasite-specific immunomodulatory functions of filarial cystatin. Infect Immun 71: 2422-9.

Smits, H. H. \& Akdis, C. A. (2014). In utero priming by worms protects against respiratory allergies. J Allergy Clin Immunol 134: $1280-1$.

Spillmann, R. K. (1975). Pulmonary ascariasis in tropical communities. Am J Trop Med Hyg 24: 791-800.

Stein, M., Greenberg, Z., Boaz, M., Handzel, Z. T., Meshesha, M. K. \& Bentwich, Z. (2016). The Role of Helminth Infection and Environment in the Development of Allergy: A Prospective Study of Newly-Arrived Ethiopian Immigrants in Israel. PLoS Negl Trop Dis 10: e0004208.

Straubinger, K., Paul, S., Prazeres da Costa, O., Ritter, M., Buch, T., Busch, D. H., Layland, L. E. \& Prazeres da Costa, C. U. (2014). Maternal immune response to helminth infection during pregnancy determines offspring susceptibility to allergic airway inflammation. J Allergy Clin Immunol 134; 1271-1279 e10. 
Takeuchi, H., Khan, A. F., Hasan, M. I., Hawlader, M. D., Yunus, M., Zaman, K., Chowdhury, H. R., Takanashi, S., Wagatsuma, Y. \& Iwata, T. (2016). Comment on IgE responses to Ascaris and mite tropomyosins are risk factors for asthma. Clin Exp Allergy 46; 178-80.

Takeuchi, H., Khan, A. F., Yunus, M., Hasan, M. I., Hawlader, M. D., Takanashi, S., Kano, H., Zaman, K., Chowdhury, H. R., Wagatsuma, Y., Nakahara, S. \& Iwata, T. (2015). Anti-Ascaris immunoglobulin E associated with bronchial hyper-reactivity in 9-year-old rural Bangladeshi children. Allergol Int.

Takeuchi, H., Zaman, K., Takahashi, J., Yunus, M., Chowdhury, H. R., Arifeen, S. E., Baqui, A., Wakai, S. \& Iwata, T. (2008). High titre of anti-Ascaris immunoglobulin E associated with bronchial asthma symptoms in 5-year-old rural Bangladeshi children. Clin Exp Allergy 38: 276-82. van den Biggelaar, A. H., van Ree, R., Rodrigues, L. C., Lell, B., Deelder, A. M., Kremsner, P. G. \& Yazdanbakhsh, M. (2000). Decreased atopy in children infected with Schistosoma haematobium: a role for parasite-induced interleukin-10. Lancet 356: 1723-7.

Zakzuk, J. (2016). Inmunorregulación inducida por helmintos: una actualización. . Iatreia 29: 182-193.

Zakzuk, J., Bornacelly, A., Mercado, D., Sánchez, J., Acevedo, N., Caraballo, L. (2014). The evolution of IgE sensitization to Ascaris allergenic components in early infancy. In Allergic diseases: from mechanisms to cure (ed. S. J. Galli, You-Young Kim), pp. 29-31. Pacini Editore: Italy.

Zakzuk, J., Jimenez, S., Cheong, N., Puerta, L., Lee, B. W., Chua, K. Y. \& Caraballo, L. (2009). Immunological characterization of a Blo t 12 isoallergen: identification of immunoglobulin E epitopes. Clin Exp Allergy 39: 608-16. 